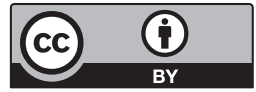

\title{
Los significados de la participación para el cambio climático en Chile*
}

\section{The Meanings of Participation for Climate Change in Chile}

\author{
Rodolfo Sapiains Arrué**, Ana María Ugarte Caviedes***, \\ Paulina Aldunce****
}

Recibido: 2017-03-10 // Aprobado: 2017-05-03// Disponible en linea: 2017-06-30

Cómo citar este artículo: Sapiains A. R., Ugarte Caviedes, A. M. y Aldunce, P. (2017). Los significados de la participación para el cambio climático en Chile. Ambiente y Desarrollo, 21(41), 43-60. https://doi.org/10.11144/Javeriana.ayd21-41.spcc

doi:10.11144/Javeriana.ayd21-41.spcc

\section{Resumen}

Este artículo analiza los distintos significados del concepto de participación, para avanzar hacia un modelo más inclusivo de gobernanza del cambio climático en Chile. Para ello, se presenta una revisión bibliográfica que discute distintas epistemologías, teorías y definiciones de la participación, con énfasis en las dificultades para su implementación en el contexto chileno. Posteriormente, se revisan los mecanismos de participación ciudadana desplegados en el desarrollo de instrumentos de gobernanza del cambio climático existentes en Chile. Se distinguen tipos de participación utilizados y se identifican experiencias conducidas desde la sociedad civil y la academia. Finalmente, se discuten los alcances y las limitaciones de los modelos de participación implementados y se resalta la importancia de incrementar la influencia de la sociedad civil y de mejorar los mecanismos existentes. Esto se explica por un escenario de cambio climático que posiblemente requerirá una mayor cantidad de actores involucrados en la toma de decisiones, para anticipar posibles divisiones frente al desarrollo de acciones de adaptación o mitigación más radicales, y que al mismo tiempo demandará mayores niveles de responsabilidad, compromiso y acción de la ciudadanía.

Palabras clave: participación; cambio climático; gobernanza; Chile; adaptación

\footnotetext{
* $\quad$ Este artículo surge del proyecto de investigación titulado Integrando el conocimiento psicológico y el estudio de las dinámicas sociales en el abordaje del cambio climático en Chile, financiado por el programa PAI-Conicyt Chile, folio \#82140012, agosto de 2015 - julio de 2017.

** Psicólogo, Ph. D. Psychological Factors for Environmental Management, The University of Queensland. Académico de la Universidad de Chile. Correo electrónico: rodolfo.sapiains@gmail.com

*** Psicóloga, doctora (candidata), Programa de Estudios Latinoamericanos, Universidad de Chile. Correo electrónico: amuc@u.uchile.cl

**** Ingeniera agrónoma, Ph. D. Social Sciences and Natural Resources Management, The University of Melbourne. Académica de la Universidad de Chile. Correo electrónico: paldunce@uchile.cl
} 


\begin{abstract}
This article analyzes the different meanings of the concept of participation, as to move towards a more inclusive model of climate change governance in Chile. For this purpose, we present a bibliographic review that discusses different epistemologies, theories, and definitions of participation, with emphasis on the difficulties for their implementation in the Chilean context. Subsequently, we review the citizen participation mechanisms deployed in the development of existing climate change governance instruments in our country. We characterize the types of participation used and identify the experiences conducted from civil society and academia. Finally, we discuss the scope and limitations of the participation models implemented and highlight the importance of increasing the influence of civil society and improving existing mechanisms. This is explained by means of a scenario of climate change that would possibly require a greater number of actors involved in decision making, as to anticipate possible divisions in the face of the development of more radical adaptation or mitigation actions, and at the same time, demand higher levels of responsibility, commitment, and action from the citizens.
\end{abstract}

Keywords: participation; climate change; governance; Chile; adaptation 


\section{Introducción}

El cambio climático puede ser entendido como un problema socioambiental complejo, de difícil solución, cuyo abordaje excede las ciencias del clima y desafía aspectos centrales de la vida moderna. Estas y otras características sugieren que el cambio climático puede ser definido como un wicked problem, categoría de problemas que son difíciles de definir, que tienen muchos componentes interdependientes y frecuentemente múltiples causas, cuyas soluciones redundan en consecuencias imprevistas; que usualmente no son estables, no tienen una sola clara solución, difícilmente son responsabilidad de una sola organización o grupo de personas; involucran cambios conductuales y se caracterizan por recurrentes fallas en las políticas públicas para enfrentarlos (Rittel y Webber, 1973).

Gupta (2016) sugiere que el cambio climático es un super wicked problem, pues enfrentarlo implica cuestionar los actuales pilares de nuestra civilización, como el libre mercado y el consumismo. Por lo tanto, es un problema que requiere una aproximación multidisciplinaria que actúe a distintos niveles sociales y cuente con la participación de la mayor cantidad de actores sociales.

El Grupo Intergubernamental de expertos sobre el Cambio Climático (IPCC) $(2013,2014)$ ha recalcado, consistentemente, que el desarrollo de estrategias de mitigación y adaptación debe considerar tanto los componentes físicos y económicos del cambio climático como su dimensión social y psicológica. Las características propias del fenómeno lo hacen un problema difícil de comunicar y múltiples barreras psicológicas y sociales influyen en cómo las personas lo perciben y responden a este. Además, se trata de un problema que presenta altos niveles de incertidumbre, en particular respecto a responsabilidades y a las acciones más adecuadas para enfrentarlo (Aldunce et al., 2016a). En este contexto, el estudio de la dimensión humana del cambio climático es fundamental, y de ahí la necesidad de una reflexión respecto al tipo de participación ciudadana que prevalece en cada sociedad y la que se necesita para abordar los múltiples desafíos que se presentan (Aldunce et al., 2016b).

En este sentido, son múltiples las críticas realizadas a las perspectivas de arriba hacia abajo (topdown) en programas y políticas ambientales, por el limitado o nulo rol atribuido a las comunidades que habitan zonas vulnerables o que son mayormente afectadas por este tipo de problemas (Berkes, 2004; Torquebiau y Taylor, 2009). Desde distintos ámbitos se ha demandado una perspectiva más integral, que incorpore la mirada de abajo hacia arriba (bottom-up) en áreas como la conservación de la biodiversidad (e. g., Kajfez Bogataj, 2009; Wilshusen, Brechin, Fortwangler y West, 2002) y el manejo de recursos naturales (e. g., Murphree, 2009; Torquebiau y Taylor, 2009).

De acuerdo con Beeton y Lynch (2012), la protección ambiental de cualquier región no es posible sin la inclusión de quienes habitan dichos territorios en amplias redes de trabajo que se orienten tanto a objetivos ambientales como a objetivos sociales. De hecho, en muchos casos, la falta de participación de las personas en programas ambientales ha conducido a bajos niveles de éxito, sin lograr la protección del medio ambiente ni mejoras en las condiciones de vida de las personas (Boissiere, Sheil, Basuki, Wan y Hien, 2009).

Esto se puede explicar por las dificultades que pueden emerger para implementar soluciones que de un modo u otro alteran los estilos de vida y patrones de comportamiento habitual en cada sociedad. Por ejemplo, en sectores rurales, la solución a muchos problemas ambientales puede demandar cambios significativos en los sistemas de vida de comunidades que dependen del uso de ecosistemas vulnerables y cuya explotación debe ser restringida o mejor administrada. En zonas urbanas, respuestas complejas a problemas ambientales pueden incluir cambios drásticos en los estilos de vida dominantes, de manera que se reduzcan significativamente los niveles de consumo energético o de bienes y servicios. Por ello, la integración de la ciudadanía en el proceso de diseño, implementación y evaluación de políticas públicas para enfrentar el problema resulta fundamental (Aldunce et al., 2016; Collins e Ison, 2009; Webb, 2012). 
En Latinoamérica, la mayoría de los gobiernos se han abierto a marcos institucionales que en general incorporan a las personas en la toma de decisiones políticas, mediante la ampliación y legitimación de espacios para la participación en la gestión pública (Villarroel y Erlwein, 2007). Sin embargo, y particularmente en Chile, diversos estudios (Ferrada, 2007; Aldunce et al., 2015; Sabatini, Sepúlveda y Blanco, 2000; Sepúlveda y Bettati, 2005) han identificado vacíos y problemas en cuanto a la participación ciudadana en la institucionalidad ambiental, razón por la cual recomiendan mejorar la cooperación público-privada, fortalecer la participación de organismos de la sociedad civil en contenidos, procedimientos, seguimiento y evaluación de políticas públicas; mejorar la base de información ambiental para la toma de decisiones; aumentar la fiscalización de normas, resoluciones y leyes y las oportunidades de ejercer el derecho ciudadano a rendiciones de cuenta por parte de autoridades, así como promover una mayor integración intersectorial en la generación e implementación de políticas. En este sentido, cobra importancia el preguntarse de qué participación estamos hablando cuando los gobiernos se abren a la integración de la ciudadanía en las políticas públicas.

Este artículo presenta una discusión sobre los significados, implicancias y limitaciones del concepto de participación, por sí solo y en relación con un modelo de gobernanza colaborativa. Describe los principales actores involucrados en el abordaje del cambio climático y analiza la participación ciudadana que ha existido en la elaboración de diversos instrumentos normativos generados por organizaciones gubernamentales para enfrentar el problema en Chile. Finalmente, discute los significados de dicha participación y cómo avanzar hacia un modelo en el que los ciudadanos tengan un rol protagónico.

\section{Sobre el concepto de participación}

Cornwall (2008) ha señalado que el uso masivo y tal vez indiscriminado del concepto de participación por múltiples organizaciones como el Banco Mundial, ONG, gobiernos e incluso empresas privadas sugiere que se trata de un concepto extremadamente maleable y susceptible de ser utilizado para obedecer a distintos intereses, pues puede significar casi cualquier cosa que involucre personas. En ese sentido, es importante hablar de una "epistemología de la participación" (Healy, 2009) que nos permita entender los diversos significados, racionalidades o posiciones teóricas desde los cuales se utiliza este concepto y entender sus consecuencias prácticas y políticas.

De acuerdo con Wandersman (1984), se pueden identificar al menos dos posiciones dominantes respecto al significado de la participación: una que apunta a un sujeto pasivo, receptor de servicios, y otra que concibe a un sujeto activo, gestor de acciones y decisiones. Así mismo, la vasta producción teórica en torno a la participación implica considerar diversos aspectos de esta: sus tipos (e. g., social, ciudadana, comunitaria, política), sus mecanismos (e. g., asambleas, mesas de trabajo, consultas), los contextos donde se desarrolla (e. g., laborales, comunitarios, educativos), las condiciones existentes (e. g., apoyo institucional, recursos), los procesos vinculados a ella (e. g., fortalecimiento, liderazgo, poder) y los valores que conlleva (e. g., equidad, justicia, inclusión, pluralidad) (Wiesenfeld y Sánchez, 2012). En este sentido, hablar de participación para el abordaje del cambio climático demanda una clara definición conceptual.

La clásica teoría sobre la participación de Arnstein (1969) define distintos niveles de esta en relación con la toma de decisiones, presentados como peldańos de una escalera que incluye siete niveles, el primero de los cuales lo conforman la manipulación y la terapia, donde no existe participación alguna. Luego se continúa con informar, consultar y apaciguar (participación simbólica), hasta llegar a los niveles reales de participación identificados como asociación, poder delegado y control ciudadano. Lo central en este modelo es la concepción del poder como algo que ciertos grupos poseen (generalmente instituciones de gobierno y grandes empresas) y que debe ser traspasado, de manera progresiva, a quienes no lo tienen (generalmente las comunidades). La participación, desde esta perspectiva, es un fin en sí misma y un medio para alcanzar mejoras sociales. 
Cornwall (2008) propone una tipología de la participación que destaca lo que esta significa tanto para la institución, organización o empresa, como para sus receptores o beneficiarios, así como los objetivos de esta, en función de lo cual se definen cuatro tipos: participación nominal, instrumental, representativa y transformativa. Esta última se orientaría al empoderamiento de las personas y las comunidades, siendo un medio y un fin para la transformación social. Otros autores, como Kumar (2006) y Healy (2009), abogan por otorgarle un rol relevante a los significados que las personas construyen sobre la participación, tanto a nivel de sentido como a nivel de valores implicados.

Plantear como eje de la participación social una perspectiva subjetiva implica un giro en la concepción tradicional, pues esta ya no es exclusivamente científica, sino que incorpora los saberes, significados y percepciones que todos los actores relacionados con la temática van construyendo y negociando. Por lo tanto, supone un enfoque de proceso que da cuenta de la dinamicidad del concepto y de la necesidad de incorporar debate y diálogo al momento de diseñar estrategias más efectivas de participación social, la cuales disminuyan las asimetrías de poder e información entre los diferentes actores.

Desde la psicología comunitaria, Sánchez (2000) define la participación como un proceso dinámico, voluntario y flexible, que se expresa en diferentes mecanismos, formas y acciones según las condiciones del contexto y el tipo de actores, donde toda acción sería acordada por los miembros de la comunidad con base en asuntos de interés común vinculados a su cotidianidad. En la misma línea, Montero (2006) propone que la participación comunitaria es un proceso organizado, colectivo, libre, incluyente, en el cual hay una variedad de actores, de actividades y de grados de compromiso, y que está orientado por valores y objetivos compartidos, en cuya consecución se producen transformaciones comunitarias e individuales.

Arango (2007) agrega que todas las acciones acordadas por la comunidad son reflexivas, guiadas por principios de cooperación, solidaridad y ayuda mutua, cuya meta es la autogestión en la solución de los problemas y la satisfacción de necesidades. Se trata, por tanto, de una forma de entender la participación que requiere no solo voluntad política por parte de quienes ocupan cargos de representación social, sino también de la misma sociedad civil, la cual debe organizarse bajo ciertos principios para hacerse cargo de esta responsabilidad (De Lellis, 2006).

Desde las ciencias sociales y políticas, esta responsabilidad de la sociedad civil se ha analizado bajo el concepto de ciudadanía, tradicionalmente entendido como la expresión de una construcción colectiva que configura las relaciones entre las personas, haciéndolas miembros de una sociedad, fundada en el respeto de un conjunto de derechos y prerrogativas por parte de los miembros de un Estado, y en la práctica de ciertas acciones jurídicas, políticas, económicas y culturales que definen la membresía social (Turner, 1993).

Con el advenimiento de la modernidad, la idea de los derechos se asocia a la libertad individual, lo que marca el surgimiento de una nueva subjetividad liberal, donde el estatus de ciudadano se relaciona con el reconocimiento formal de la integración de un individuo en el sistema de derechos y deberes civiles, políticos y sociales que garanticen tanto esta libertad como la posibilidad de elegir y ser elegido, así como la seguridad y el bienestar económico necesarios para una adecuada cohesión social.

Para Sandoval (2003), este discurso de ciudadanía propone la promoción de una imagen restringida y sectorial del sujeto titular de derechos: un individuo varón y occidental, urbano, heterosexual y adulto, e invisibiliza o incluso reprime otras formas de subjetividad. A este respecto, Lechner (2007) propone que la relación sujeto-sociedad en el marco de la ciudadanía tendría un carácter históricocultural, transformándose en el transcurrir del tiempo, respondiendo a estructuras sociales diversas.

Si la noción de ciudadanía es dinámica, lo que tradicionalmente ha sido definido como tal no es más que un imaginario moderno, por lo tanto, no es la única forma de constituir o entender la ciudadanía; es decir, la idea de responsabilidad civil en torno a ciertos derechos no es la única manera 
de participar en la sociedad. Es necesario incorporar marcos comprensivos que permitan entender la ciudadanía y la participación social desde la emergencia de nuevas o renovadas subjetividades, para ampliar así las posibilidades de participación de las personas en los procesos de transformación social (Tejerina, 2005).

Para Prilleltensky (2003), siguiendo esta propuesta, la participación social se constituye en un componente y a la vez en una condición para el bienestar social. En la misma línea, Rodríguez (2002) sostiene que la participación es un medio para enfrentar las condiciones de vulnerabilidad social propias del contexto latinoamericano, pues al fomentar el control de los actores sobre su entorno aumenta la percepción de eficiencia de su accionar y se producen cambios sociales de mayor impacto. En este sentido, es también un proceso de fortalecimiento del capital social de las poblaciones (Leiva, 2003; Aldunce et al., 2016b).

Por lo tanto, como sostienen Rivera, Velázquez y Morote (2014), la participación no es solo la expresión de las necesidades, opiniones e ideas, sino que se centra en el poder de los sujetos en la toma de decisiones, y debe contribuir a la autovaloración y reflexión en torno a la vida cotidiana, otorgando a las personas la posibilidad de crear y recrear nuevas formas de vida y convivencia. La participación sería uno de los pilares para el cambio social, y concebirlo así implica rescatar las ventajas políticas del proceso, pues la organización, los desarrollos y las acciones comunitarias se presentan como una demanda de mayor injerencia por parte de los ciudadanos en la gestión gubernamental.

\section{Gobernanza y participación}

Una manera de situar el concepto de participación y su significado es enmarcándolo dentro del modelo de gobernanza colaborativa, acuerdo gubernamental donde una o más agencias públicas comprometen directamente a actores no gubernamentales en un proceso de toma de decisiones colectivo que es formal, orientado al consenso y deliberativo y que apunta a diseñar o implementar políticas públicas o a gestionar programas o recursos públicos (Ansell y Gash, 2008).

En el caso del cambio climático en Chile, el modelo de gobernanza colaborativa que fomenta la inclusión de la ciudadanía en la toma de decisiones resulta no solo pertinente, sino necesario, considerando que, como señala Barton (2013), en el país han prevalecido esquemas de gobernanza no inclusivos, sectoriales y fragmentados. Barton, Krellenberg y Harris (2014) recalcan que la gobernanza colaborativa incrementa la legitimidad del proceso y sus resultados, y mejora el abordaje de los complejos impactos del cambio climático, posibilitando la inclusión de una gran cantidad de actores.

No obstante, se han identificado múltiples dificultades y barreras para implementar un sistema de gobernanza que propicie escalar la participación ciudadana para el cambio climático en Chile (Aldunce et al., 2015; Barton, 2013). Estas se relacionan con la falta de experiencia en Chile con este tipo de enfoques, falta de cultura participativa, cambios en el entorno institucional, visión de corto plazo, limitado financiamiento y capital humano para efectuar este proceso, y escaso interés y voluntad política de muchas autoridades para implementarlo. Esto es reforzado por una estructura institucional autoritaria y asistencialista y por una cultura de la pasividad, ambas herencias de la dictadura militar de 1973-1990 (Bornand, 2010).

En este sentido, comprender las dificultades de la participación ciudadana en el contexto chileno implica considerar que el orden democrático postdictatorial se ha caracterizado por fomentar una acción política pasiva y por contener un conjunto de creencias y valores negativos sobre la participación que la transforman en un tema irrelevante, remitido a la esfera individual y doméstica.

En Chile la cultura política de la pasividad puede observarse en la actual mirada técnica y despolitizada sobre las demandas sociales, lo que configura acciones aparentemente colectivas, que, en estricto rigor, apuntan principalmente a la autorrealización y a la satisfacción individual. De hecho, 
la Tercera Encuesta Nacional de Medio Ambiente y Cambio Climático muestra que menos del 5\% de la población ha participado en actividades relacionadas con temáticas ambientales en general o relacionadas con el cambio climático en particular (i. e., consultas públicas, talleres educativos, mesas de trabajo, marchas, voluntariado), y alrededor de un $45 \%$ no está siquiera interesado en participar (Sapiains, Ugarte y Rdz-Navarro, 2017).

\section{Gobernanza del cambio climático}

En el caso del cambio climático, la discusión anterior sugiere que, sin un marco de gobernanza apropiado, hay riesgo de que se desarrolle una respuesta superficial, puramente infraestructural y con una implementación deficiente, en lugar de una respuesta intersectorial con base comunitaria, integrada en una conciencia más colectiva sobre el problema y que posibilite las transformaciones sociales y culturales necesarias para enfrentarlo.

Collins e Ison (2009) plantean una epistemología del poder más relacional, que permita tanto democratizar estos procesos como abordar colaborativamente los desafíos del cambio climático, lo cual implica reconocer que alcanzar completa participación no es la única manera válida, necesaria o posible para abordar el problema. Los autores critican el modelo de participación de Arnstein, pues limita u omite el valor de otros tipos de participación, si se considera que para muchas personas alcanzar niveles de influencia más altos no es un fin en sí mismo, sino un medio para alcanzar ciertos objetivos, y que, en general, no todas las personas quieren participar, o en muchos casos formas alternativas no institucionales de participación tienen una importancia fundamental. Al mismo tiempo, en estos modelos lineales un foco exclusivo en el traspaso del poder omite que los problemas también cambian, lo que además suele ocurrir cuando más personas participan, y que no todos los problemas requieren el mismo nivel de participación. Finalmente, el contexto donde las relaciones de poder y el problema suceden tampoco puede ser omitido.

En una perspectiva similar, Gupta (2016) cuestiona que siempre sean necesarios altos niveles de participación de la sociedad civil en el abordaje del cambio climático. Haciendo un análisis sobre la evolución histórica del cambio climático como problema social y político a nivel global, la autora plantea que el nivel de participación depende de al menos dos factores: el grado de consenso en torno al problema y sus soluciones y los niveles de confianza entre los actores. Sugiere así que mientras el problema permaneció con altos niveles de consenso (años noventa), con confianza entre las partes y bien estructurado, la participación de otros actores (no expertos ni del ámbito de las políticas públicas) no fue tan necesaria como lo es actualmente, pues se trataba de un problema técnico cuyas soluciones eran relativamente claras y respaldadas por la mayoría de los actores.

En la medida que se hizo evidente que el abordaje del cambio climático implicaría transformaciones sociales radicales, el consenso sobre el problema comenzó a decaer, pasó de ser un problema técnico altamente estructurado, a constituir un complejo problema social, ambiental y finalmente político, con altos niveles de desconfianza entre las partes involucradas y altamente desestructurado. Gupta (2016) sostiene que, en este contexto, se requiere una mayor participación, con el objetivo de volver a transformar el cambio climático en un problema estructurado, con altos niveles de confianza entre las partes y que genere consenso. De lo anterior se desprende que la participación es un proceso dinámico, relacional y contextual, cuya intensidad y características dependen de múltiples factores.

En esta línea, Collins e Ison (2009) proponen un modelo de participación orientado hacia el aprendizaje social, basado en una epistemología de la participación que sostiene que el conocimiento ocurre al construir un problema entre todos los actores involucrados y al buscar formas de enfrentarlo colaborativamente. Esto implica un proceso en el cual resulta clave facilitar que los actores reconozcan la diversidad de visiones de mundo y comprendan que el problema tiene dimensiones sociales y biofísicas donde es necesario desarrollar habilidades para la comunicación, el aprendizaje social y la acción concertada, reconociendo la existencia y el valor de un modelo distinto a los tradicionales enfoques economicistas y biofísicos para enfrentar el cambio climático. 
En este sentido, un modelo de gobernanza colaborativa para el cambio climático se puede definir como un proceso de aprendizaje social que puede ser profundizado superando la diferencia entre las limitaciones de la ciencia (proyecciones a largo plazo y con un alto grado de incertidumbre) y los objetivos políticos de corto plazo, en conjunto con perspectivas sectoriales y donde la participación de múltiples actores es fundamental para el desarrollo de estrategias más efectivas y duraderas, con acceso a un mayor pool de alternativas de solución y con un enfoque más proactivo que reactivo a eventos climáticos específicos (Amundsen, Berglund y Westskog, 2010).

Esto implica la incorporación de instituciones públicas y otros actores, principalmente la academia, la sociedad civil y el sector privado, dentro de un modelo de gobernanza complejo y transversal, donde el cambio climático esté vinculado a temáticas del desarrollo en un sentido más amplio, incluyendo, por ejemplo, aspectos relacionados con la vulnerabilidad social, modos de transporte, asentamientos, vivienda, gestión del agua y la energía y uso de suelos, entre otros.

En este contexto, las personas como sujetos individuales u organizadas en comunidades son un actor principal. Sus percepciones, valores y normas pueden facilitar o inhibir su acción y con ello promover o limitar la mitigación y la adaptación, pues son precisamente estos elementos los que sostienen sus visiones de mundo y estilos de vida. Más aún, dada la relevancia de este actor social, su injerencia en los procesos de diseño, ejecución y evaluación de estrategias, políticas y programas en relación con el cambio climático desarrollados en Chile no es clara ni suficiente. Esto puede explicar que las personas, en general, perciban la problemática como relevante, pero le resten importancia comparativamente con otros asuntos públicos, lo que disminuye su intención de participar (Sapiains, Ugarte y Rdz-Navarro, 2017).

Es necesario entonces explorar si los actuales modelos de gobernanza y participación son adecuados para enfrentar los desafíos ambientales actuales y los escenarios futuros para Chile. La efectividad de las acciones para abordar el cambio climático va a depender tanto de la voluntad política como de la aceptación social de dichas medidas y del involucramiento de la ciudadanía, así como de los costos que podría implicar para el país y para las personas en el corto, mediano y largo plazo. Desde este punto de vista, se considera que el desarrollo de la gobernanza es tan importante como las políticas y la gestión públicas para mitigar y adaptarnos al cambio climático (Adger, 2003).

Para profundizar en este análisis, a continuación se revisa cómo se han desarrollado en Chile las principales estrategias, proyectos, planes y programas gubernamentales para enfrentar el problema, identificando los niveles y tipos de participación que la ciudadanía ha tenido en las distintas fases de la política pública. Después se discute cuál debiese ser el tipo de participación adecuado para abordar las complejidades del cambio climático. Finalmente, se analiza en profundidad cuáles son los desafíos para la implementación de un modelo de gobernanza colaborativa para el cambio climático, considerando las particularidades del contexto chileno.

\section{Participación ciudadana en el abordaje del cambio climático en Chile}

Se puede afirmar, asumiendo las complejidades antes mencionadas, que la participación ciudadana involucra y emerge, principalmente, desde cuatro tipos de actores: agencias de gobierno, sociedad civil, academia y sector privado. Como se señaló antes, en el contexto del cambio climático, han sido las agencias de gobierno las encargadas de promover y articular las distintas instancias de participación para el desarrollo de instrumentos formales (estrategias, planes y programas gubernamentales). En este sentido, también es importante destacar que el Estado ha realizado un importante esfuerzo por incluir la opinión de distintos actores sociales, lo que se refleja en el ámbito de las políticas públicas en general, por ejemplo, con la Ley 20.500 sobre participación ciudadana (Gobierno de Chile, 2011), y en relación con temáticas ambientales en particular, como con la Ley 19.300 de medio ambiente (Gobierno de Chile, 2011). 5

$5 \quad$ Esta ley fue publicada originalmente en el año 1994; sin embargo, en el 2010 se realizaron modificaciones importantes, entre ellas, se creó el Ministerio, el Servicio de Evaluación Ambiental y la Superintendencia del Medio Ambiente. 
En esta sección se identifican los principales instrumentos en el dominio de la política pública de cambio climático y se exploran los procesos de participación ciudadana en cada uno de ellos. Por último, y aunque no es el objetivo de este estudio, se presenta brevemente quiénes y cómo se ha concretado la participación ciudadana que ha emergido desde otros actores sociales para enfrentar el cambio climático en nuestro país.

\section{Participación ciudadana desde las instituciones de gobierno}

En Chile se pueden identificar quince instrumentos formales para abordar el cambio climático, el primero de los cuales se publicó en el año 2006. El resto se encuentra en distintas etapas, ya sea de elaboración, aprobación, publicación o implementación (véase tabla 1). Es relevante observar que doce de estos instrumentos han contado, o está planificado que contarán, con un proceso de participación ciudadana, la cual se concretiza a través de dos canales principales: 1) talleres realizados tanto en regiones como en la capital, donde personal de la Oficina de Cambio Climático (OCC, actualmente Departamento de Cambio Climático) presenta el instrumento y recoge comentarios o impresiones de los asistentes a los talleres; y 2) procesos de consulta ciudadana sobre el instrumento, en los cuales todas las personas interesadas cuentan con un plazo para enviar sus comentarios, a través de una página web especialmente diseńada a estos efectos, por medio de la oficina de partes del Ministerio del Medio Ambiente (MMA), o por correo.

Cabe señalar que existe un mecanismo de trazabilidad de intención de incluir las observaciones recibidas en los documentos definitivos de los instrumentos, lo cual se concretiza a través de un informe que prepara el MMA, en el que cada observación recibida es acompañada de una respuesta que indica si la observación es considerada para su inclusión o no, junto a una respuesta argumentativa. El nivel de participación logrado en los distintos instrumentos del Gobierno es disímil, variando de 95 a 566 observaciones recibidas y de 13 a 242 personas que enviaron observaciones. Lo anterior, acompańado de talleres de presentación y discusión del anteproyecto que varían de uno a dos en Santiago y de tres a ocho en regiones (véase tabla 1).

El caso que denota una marcada diferencia en términos de haber alcanzado los mayores niveles de participación es el Plan de Adaptación del Sector Silvoagropecuario, en el cual el número de observaciones recibidas fue de 566, realizadas por 242 personas. Una posible explicación a esto se puede encontrar en el mayor número de talleres llevados a cabo (10), los cuales tuvieron una mayor asistencia (310 personas) en comparación con los demás instrumentos analizados.

Al respecto, es importante considerar que el proceso de elaboración de estos instrumentos, en términos de coordinación y redacción, es responsabilidad de la OCC. En la mayoría de los casos, la construcción de los instrumentos se ha realizado con base en estudios licitados por la OCC, los cuales han sido llevados a cabo por distintas organizaciones, como, por ejemplo, centros de investigación y universidades. No obstante, el hecho de que una organización distinta a la OCC haya generado un documento base para la posterior elaboración de estos instrumentos, no constituye necesariamente una participación de la academia, ${ }^{6}$ puesto que, en general, cada estudio fue llevado a cabo solo por una de estas organizaciones y no por todas las instancias académicas con competencias en cambio climático.

Por otro lado, en algunos casos hubo participación de otros actores en la elaboración de estos documentos base, como, por ejemplo, en la propuesta del PANCC 2017-2022 (Aldunce et al., 2015b) que contó con la colaboración de especialistas — académicos de distintas universidades y profesionales de la consultora Poch Ambiental y de la ONG Adapt-Chile-, lo cual permitió incluir diversas perspectivas desde el inicio del proceso, asunto relevante pues generalmente se tiende a incorporar la mirada de otros actores solo hacia el final del proceso.

$6 \quad$ En taller realizado en la ciudad de Santiago para la presentación del Plan Nacional de Adaptación, personal de la OCC señaló como participación de la academia el hecho de que una universidad había realizado la consultoría para la generación del instrumento base. 


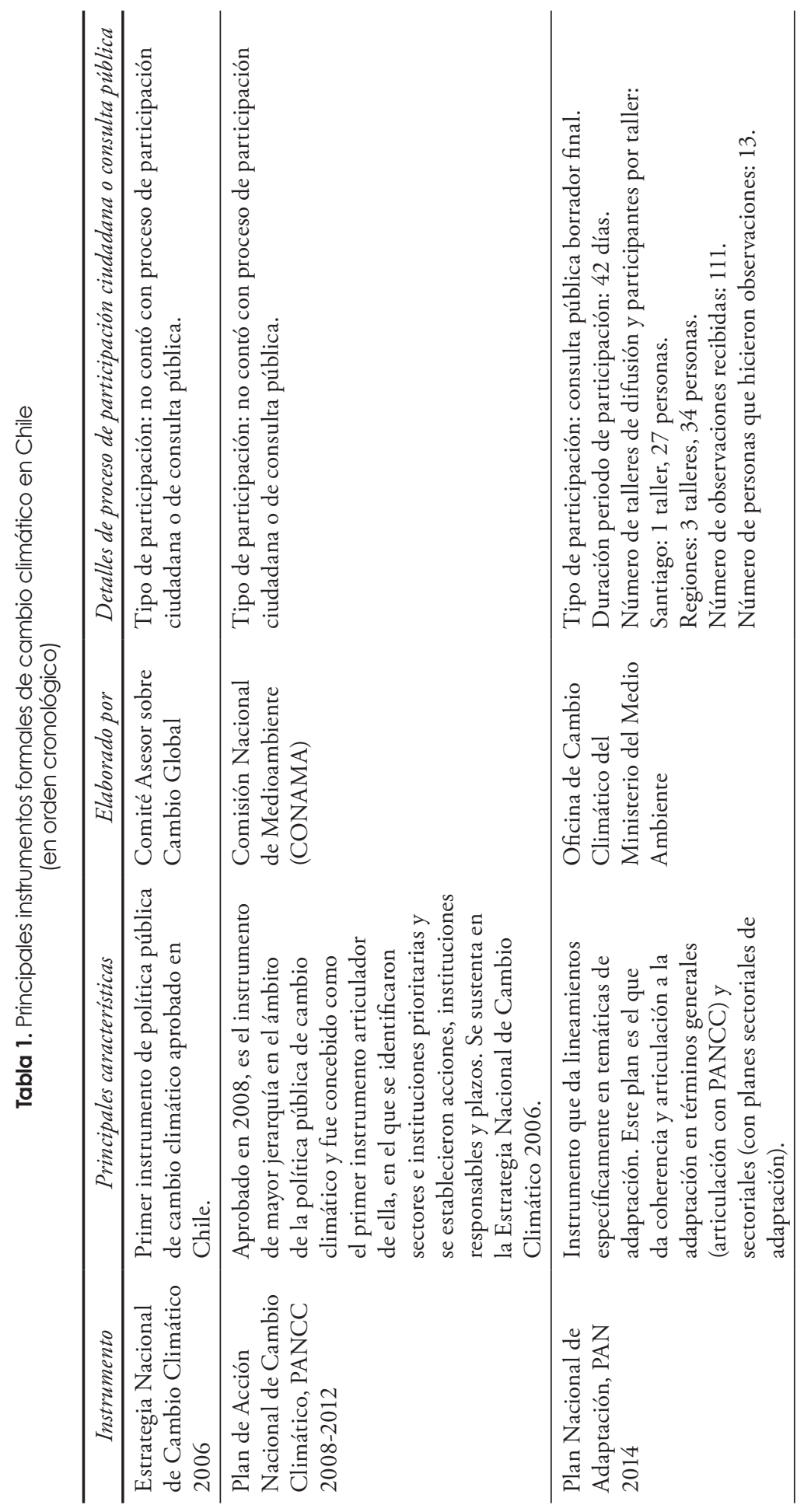




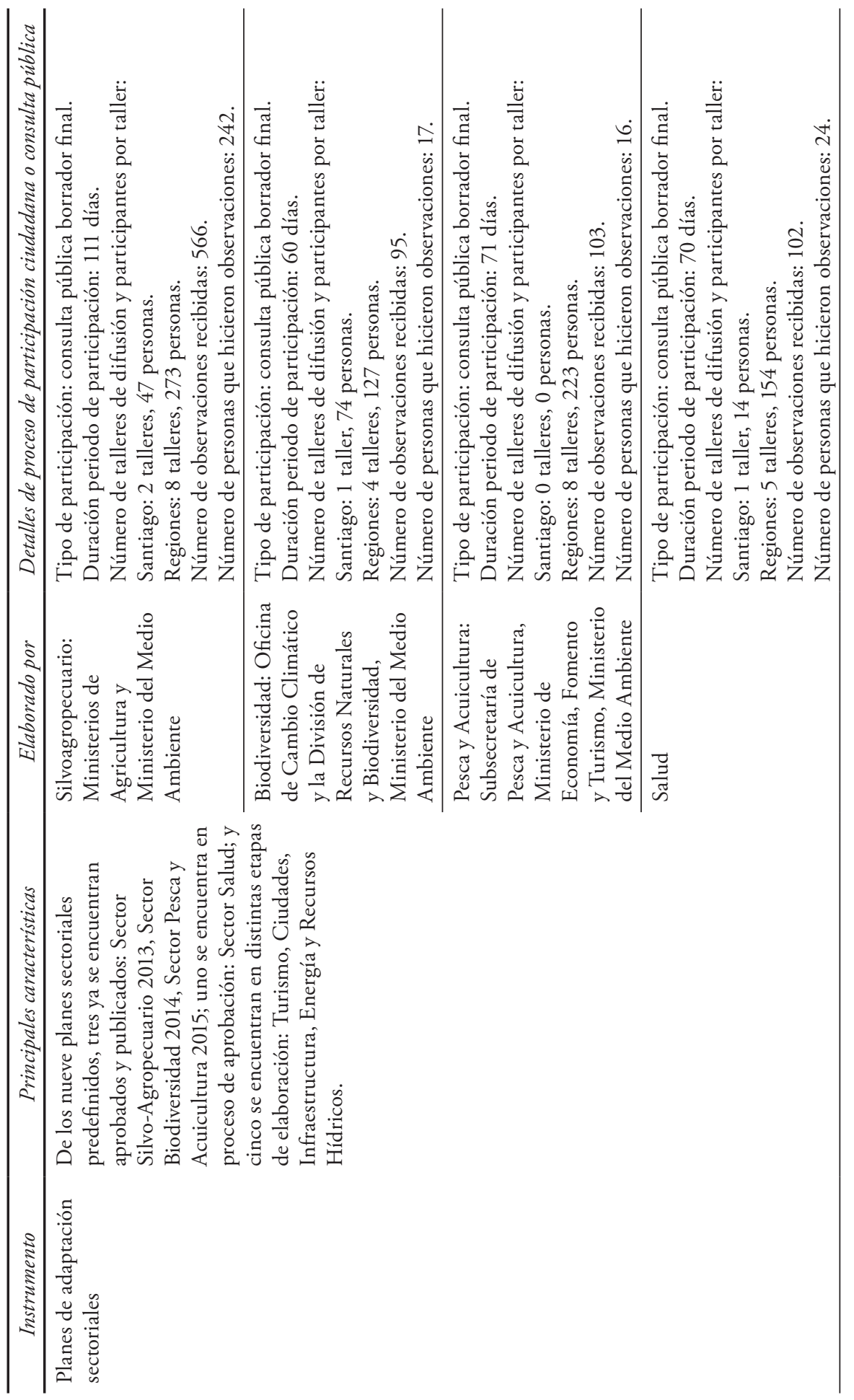




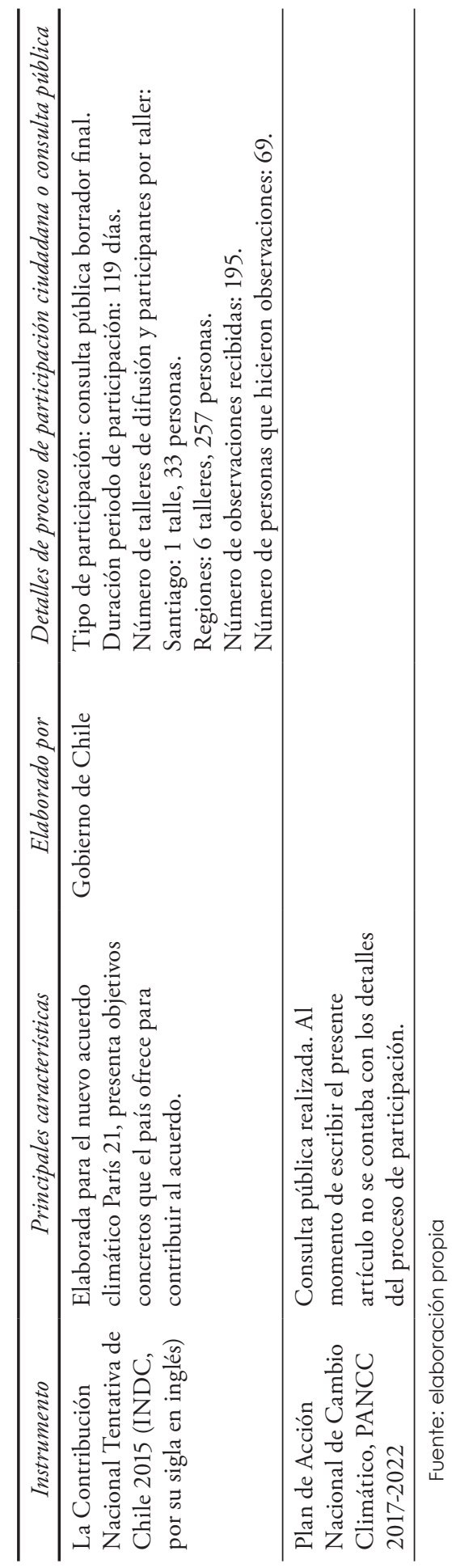


De manera complementaria, el estudio se realizó bajo un enfoque interdisciplinario, en el que se incluyeron distintas áreas que se necesitaban para sustentar un resultado óptimo a través de un balance temático de disciplinas biofísicas y sociales. El diseño del plan también contó con participación de la ciudadanía (puntos focales de los distintos ministerios, ${ }^{7}$ sector privado, población civil), a través de consulta a panel de expertos, encuestas, entrevistas a informantes claves, talleres y grupos focales. El trabajo realizado por el equipo consultor para la propuesta del plan, en colaboración con distintos actores sociales, generó los insumos necesarios de aspectos y contenidos que deberían ser considerados en el corto, mediano y largo plazo, los cuales fueron incluidos en la propuesta de contenidos (Aldunce et al., 2015b). Cabe seńalar que, si bien dicha propuesta fue utilizada como base por la OCC para la elaboración del PANCC 2017-2022, el documento final sufrió modificaciones.

\section{Participación ciudadana desde otros actores sociales}

En Chile, la participación ciudadana en temas de cambio climático no solo ha emergido desde organismos de gobierno, sino que ha sido promovida también por la sociedad civil organizada y la academia. Con respecto a la primera, la instancia más relevante es la Mesa Ciudadana de Cambio Climático, fundada en 2014 por Fundación Terram y que convoca a veinte ONG de nuestro país. Esta mesa organiza instancias de participación de diversa índole, como respuesta a los procesos de consulta ciudadana generados desde el MMA, talleres y seminarios, activismo y exhibiciones fotográficas, entre otros, y tuvo una activa participación en la COP21 realizada en París.

Otra experiencia de gran relevancia en este ámbito es el trabajo que desde el año 2013 realiza la ONG Adapt-Chile, a través de la promoción de respuestas locales (nivel municipal) al cambio climático. Esto se lleva a cabo mediante la integración de la temática como eje transversal en la toma de decisiones locales, a través de programas de trabajo que promueven la comunicación y la colaboración entre la academia y los tomadores de decisión a distintas escalas de la gestión institucional y apoyando a los actores locales con metodologías y herramientas de diagnóstico y planificación estratégica en gestión de riesgos. Junto con esto, diseña y formula propuestas de políticas públicas para avanzar hacia una sociedad resiliente. ${ }^{8}$ Entre los principales resultados de su trabajo se encuentra la Propuesta de Marco Legal e Institucional para Abordar el Cambio Climático en Chile, ${ }^{9}$ así como la coordinación de la Red Chilena de Municipios ante el Cambio Climático, instancia de cooperación, capacitación e intercambio de experiencias en torno al tema.

Por otra parte, y desde la academia, existen varios centros de excelencia que persiguen no solo la generación de conocimiento científico, sino también contribuir a la política pública a través de la colaboración para fortalecer la interfase ciencia-política-sociedad. Es el caso del Centro de Ciencias del Clima y la Resiliencia (CR2) y del Centro de Cambio Global. Ejemplo de actividades realizadas por ellos son las respuestas a consultas públicas emanadas del MMA y la organización de seminarios. En especial, se han desarrollado proyectos de investigación enmarcados en el paradigma de la investigación-acción, que buscan promover la participación ciudadana a través de la creación de plataformas de aprendizaje social (Aldunce et al., 2016b).

7 Los puntos focales de los ministerios son profesionales responsables de la temática de cambio climático en aquellos ministerios con competencia en cambio climático.

8 Véase http://www.adapt-chile.org/

9 Este proyecto, cuyo objetivo fue presentar legislación comparada sobre cambio climático y reflexionar sobre los insumos y contenidos básicos para una eventual ley de esta naturaleza en nuestro marco jurídico, fue desarrollado por Adapt-Chile junto con el Centro de Ciencia del Clima y Resiliencia (CR)2 y contó con el apoyo de la Embajada Británica en Chile y el patrocinio del Ministerio de Medio Ambiente de Chile (véase http://www.adapt-chile.org/). 


\section{Discusión}

Los documentos revisados permiten analizar la participación ciudadana, tanto en términos cuantitativos como con respecto a la epistemología de esta participación. Cuantitativamente, se observa que ha habido un esfuerzo no solo por incrementar la participación en estas actividades, sino también por descentralizar los procesos de consulta (véase tabla 1), y se ha logrado una balanceada participación entre regiones y la capital, con un lapso de participación que ha variado entre distintos instrumentos de 42 a 119 días. No obstante, la evidencia muestra que los procesos de participación ciudadana implementados tienden a concentrarse en la entrega de información y la organización de talleres y consultas públicas (principalmente a través de internet).

Si bien todas estas actividades son necesarias y muy importantes, la literatura sugiere que no son suficientes ni las más adecuadas para favorecer altos niveles de participación y compromiso ciudadano, considerando la magnitud del problema y las dificultades para la participación ciudadana en Chile. Si se aplican los modelos de participación lineales, la entrega de información a través de diversas fuentes, la organización de talleres y las consultas públicas corresponden a niveles de participación simbólicos, nominales o instrumentales, en los cuales la influencia efectiva de la ciudadanía es reducida; más aún, considerando que en la mayoría de los casos estas actividades se desarrollan solo al final del proceso, cuando los instrumentos ya están elaborados, y no en las etapas tempranas de su diseño.

Por otra parte, para modelos de gobernanza que incluyen aprendizaje social, este tipo de actividades no son suficientes para favorecer un abordaje colaborativo del cambio climático, donde todos los actores puedan dialogar para construir el problema, sus soluciones y definir sus roles en este complejo escenario social.

Esto es consistente con la evaluación del PANCC 2008-2012 (Aldunce et al., 2015), donde se concluye que existe una baja interacción de organizaciones del sector público con organizaciones de la sociedad civil y del sector privado, y el sector académico es el que tiene mayor influencia o interacción con lo institucional. Al mismo tiempo, dicha evaluación también recalca la importancia de la sensibilización de la ciudadanía con este tema y la necesidad de favorecer un mayor involucramiento de la sociedad civil, de manera que se fomenten procesos participativos.

Sin desconocer que se ha hecho un importante esfuerzo por aumentar la participación ciudadana en el tema, sobre todo considerando el contexto político, cultural e histórico del país, la literatura sugiere que es necesario avanzar hacia un tipo de participación más profunda y cualitativamente distinta a la que ha prevalecido hasta hoy. Siguiendo a Gupta (2016), se puede decir que en Chile el cambio climático aún no es un tema que genere altos niveles de desconfianza entre los actores involucrados, pues existe un consenso respecto a su importancia y está relativamente estructurado como problema científico-técnico (Sapiains, Ugarte y Rdz-Navarro, 2017).

En este sentido, se podría entender que el problema haya sido hasta ahora un tema principalmente de expertos, donde la participación más importante ha sido la de los científicos y la del gobierno de turno. Sin embargo, es probable que en la medida en que tengan que ser implementadas decisiones más radicales (p. ej., cambios en la matriz energética, patrones de consumo, impuestos a emisiones, entre otras), el aparente consenso con respecto al cambio climático comience a dar paso a discusiones y cuestionamientos que redunden en su politización, desestructuración y altos niveles de desconfianza entre los actores, todo lo cual podría replicar o acercarse a patrones de polarización ideológica observados en países como Australia o los Estados Unidos de América, con consecuencias negativas para el desarrollo de planes de mitigación y de adaptación. Esto refuerza la necesidad de incrementar los niveles de participación ciudadana y de redefinir la epistemología de la participación que se necesita.

En este sentido, se considera que la participación en el abordaje del cambio climático debería ser entendida como un proceso que puede tomar múltiples formas y acciones, en el que pueden participar todos los sectores de la sociedad, en tanto que la ciudadanía y las comunidades locales desempeñan 
un rol fundamental en todos los niveles de la política pública, no solo al final o en la implementación de acciones; donde la calidad y la profundidad de la participación dependen tanto de las condiciones institucionales que la facilitan como de la responsabilidad y el compromiso de las personas para involucrarse y utilizar dichos espacios, lo cual implica un proceso de aprendizaje en el que interactúan saberes locales y científicos, con objetivos sociales y ambientales, de corto, mediano y largo plazo, orientado a alcanzar acuerdos para lograr la transformación social necesaria para enfrentar los complejos desafíos del cambio climático.

Esta definición es consistente con un modelo de gobernanza basado en el aprendizaje social (Collins e Ison, 2009), el cual da un valor fundamental a los procesos de cambio. En estos, los problemas y las soluciones son construidos y redefinidos por múltiples actores, en un proceso de participación colaborativa donde el contexto político, histórico y social es fundamental para entender y analizar el tipo de participación que se requiere en cada caso y, asimismo, las responsabilidades de cada actor son coconstruidas en el proceso de colaboración y no previamente identificadas. Finalmente, se asume una diversidad de visiones de la realidad o epistemologías que cohabitan en la sociedad. Así, se constituye en un enfoque práctico que complementa la mirada biofísica.

La implementación de un modelo de gobernanza de este tipo demanda no solo crear condiciones institucionales que promuevan y favorezcan este tipo de participación, sino también que la ciudadanía y las comunidades locales utilicen activamente dichos espacios. En este sentido, entender la participación como un proceso de aprendizaje social (Collins e Ison, 2009) supone reconocer el desafío colectivo que implica el cambio climático, lo cual incluye y excede el ámbito institucional. Esto involucra, por ejemplo, que la responsabilidad por incrementar la participación es compartida y no puede recaer solo en las instituciones de gobierno. Por ello, es importante el trabajo que desde distintos ámbitos se puede realizar para incrementar el interés de las personas en el problema.

Además, en línea con recomendaciones de organismos internacionales como la International Association for Public Participation (IAP2), en términos de propuestas para mejorar la incorporación de la ciudadanía en el diseño, la ejecución y la evaluación de estrategias de mitigación y adaptación al cambio climático, se plantea que es necesario que los gobiernos consideren que los ciudadanos deben tener voz en toda decisión que afecte sus vidas, que sus opiniones deben influir sobre las decisiones políticas, que el proceso de participación social debe incorporar a todos los actores y otorgarles instancias para que definan cómo quieren participar, y que se realice una retroalimentación sobre el impacto que las contribuciones de los ciudadanos tienen en las decisiones políticas (Haley, 2009).

Por último, es particularmente importante el involucramiento de las comunidades que resultarán más afectadas por los impactos del cambio climático, como las poblaciones indígenas y campesinas, los adultos mayores, las mujeres y los niños y las personas que habitan zonas costeras y viven de la pesca (IPCC, 2014).

\section{Conclusiones}

El objetivo de este artículo ha sido discutir la epistemología de la participación necesaria para abordar el cambio climático en Chile. Se revisaron diversos modelos y definiciones del concepto, los cuales fueron aplicados para entender el tipo de participación ciudadana que ha prevalecido en la política pública de cambio climático en este país. Se concluye que un enfoque basado en la entrega de información, talleres y consultas llevadas a cabo principalmente por internet respondió a cómo la sociedad chilena ha interactuado con el cambio climático como un problema técnico-científico con niveles de consenso relativamente altos. No obstante, la necesidad de plantear transformaciones más radicales en la sociedad va a demandar una epistemología de la participación distinta.

En este contexto, podemos plantear que los niveles de participación ciudadana analizados siguen un proceso progresivo que debería desembocar en mayores niveles de influencia ciudadana y en una 
descentralización de la participación. En este sentido, se debería avanzar hacia un modelo de gobernanza del cambio climático que favorezca el diálogo para la coconstrucción del problema y de las futuras estrategias de acción entre todos los actores involucrados. Futuros estudios pueden desarrollar este tipo de modelos, por ejemplo, en la línea del aprendizaje social, lo que podría contribuir a entender más claramente el tipo de participación vigente y mejorar los procedimientos participativos futuros.

\section{Referencias}

Adger, W. N. (2003). Social capital, collective action, and adaptation to climate change. Economic Geography, 79(4), 387-404.

Aldunce, P., Araya, D., Lillo, G., Maldonado, P. y Ramos, I. (2016). Sequía, Valle de Aconcagua. En Prácticas de adaptación de cambio climático, informe de resultados. Santiago de Chile: CR2.

Aldunce, P., Beilin, R., Handmer, J. y Howden, M. (2016a). Is climate change framed as "business as usual” or as a challenging issue? The practitioners'dilema. Environment y Planning C: Government y Policy, 34(5), 999-1019. doi: 10.1177/0263774X15614734. 00.

Aldunce, P., Beilin, R., Handmer, J. y Howden, M. (2016b). Stakeholder participation in building resilience to disasters in a changing Climate. Environmental Hazards, 15(1), 58-73.

Aldunce, P., Bello, F., Bórquez, R., Farah, M., Echeverría, I., Indvik, K. y Rudnick, A. (2015). Evaluación de término del Plan de Acción Nacional de Cambio Climático Ministerio del Medio Ambiente, Gobierno de Chile. Santiago de Chile: Ministerio del Medio Ambiente.

Amundsen, H., Berglund, F. y Westskog, H. (2010). Overcoming barriers to climate change adaptation - A question of multilevel governance Environment and Planning C: Politics and Space, (28), 276-289. doi:10.1068/c0941.

Ansell, C. y Gash, A. (2008). Collaborative governance in theory and practice. Journal of Public Administration Research and Theory, 18(4), 543-571. doi: 10.1093/jopart/mum032

Arango, C. (2007). Psicología comunitaria de la convivencia. Cali: Universidad del Valle.

Arnstein, S. (1969). A ladder of citizen participation. Journal of the American Planning Association, 35(4), 216-214.

Barton, J. (2013). Climate change adaptive capacity in Santiago de Chile: creating a governance regime for sustainability planning. International Journal of Urban and Regional Research, 37(6), 1916-1933. doi: $10.1111 / 1468-2427.12033$.

Barton, J., Krellenberg, K. y Harris, J. (2014). Collaborative governance and the challenges of participatory climate change adaptation planning in Santiago de Chile. Climate and Development, 7(2), 175-184. doi: 10.1080/17565529.2014.934773.

Beeton, R. y Lynch, J. (2012). Most of nature: a framework to resolve the twin dilemmas of the decline of nature and rural communities. Environmental Science and Policy, (23), 45-56.

Berkes, F. (2004). Rethinking community-based conservation. Conservation Biology, 18(4), 621-630.

Boissiere, M., Sheil, D., Basuki, I., Wan, M. y Hien, L. (2009). Can engaging local people's interests reduce forest degradation in Central Vietnam? Biodiversity Conservation, (18), 2743-2757.

Bornand, M. (2010). Educación para la ciudadanía. Sobre la formación y emergencia del sujeto político en la escuela. Documento presentado en el Congreso Iberoamericano de Educación METAS 2021, Argentina [online]. Recuperado el 12 de octubre de 2016, de http:/www.adeepra.org.ar/congresos/Congreso 
IBEROAMERICANO/EDUCCIUDADANIA/R0597_Bornand.pdf

Collins, K. e Ison, R. (2009). Jumping off Arnstein's ladder: social learning as a new policy paradigm for climate change adaptation. Environmental Policy and Governance, (19), 358-373.

Cornwall, A. (2008). Unpacking "participation": models, meanings and practices. Community Development Journal, 43(3), 269-283.

De Lellis, M. (2006). La psicología comunitaria en el ciclo de las políticas públicas. En M. De Lellis (Ed.), Psicología y políticas públicas de salud (pp. 125-186). Buenos Aires: Paidós.

Ferrada, N. (2007). Informe sobre institucionalidad ambiental. Santiago de Chile: Equipo de Derecho Público, Gobierno de Chile.

Gobierno de Chile. (2011a). Ley 20.500 sobre Asociaciones y Participación Ciudadana en la Gestión Pública. Santiago de Chile: autor.

Gobierno de Chile. (2011b). Ley 19.300 sobre Bases Generales del Medio Ambiente. Santiago de Chile: autor.

Gupta, J. (2016). Climate change governance: history, future, and triple-loop learning? Wiley Interdisciplinary Reviews: Climate Change, 7(2), 192-210. doi: 10.1002/wcc.388.

Healy, S. (2009). Toward an epistemology of public participation. Journal of Environmental Management, (90), 1644-1654.

Intergovernmental Panel on Climate Change (IPCC). (2013). Managing the risks of extreme events and disasters to advance climate change adaptation. A special report of working groups $i$ and ii of the Intergovernmental Panel on Climate Change. Nueva York: Cambridge University Press.

Intergovernmental Panel on Climate Change (IPCC). (2014). Climate Change 2014: Synthesis report. Contribution of working groups I, II and III to the fifth assessment report of the Intergovernmental Panel on Climate Change. Ginebra: autor.

Kajfez Bogataj, L. (2009). Climate change and future adaptation. Economic and Business Review, 11(1), 9-27.

Kumar, A. (2006). Creative social research: rethinking theories and methods and the calling of an ontological epistemology of participation. Dialectical Anthropology, (30), 227-271.

Lechner, N. (2007). Los desafíos políticos del cambio cultural. En N. Lechner (Ed.), Obras escogidas (pp. 39-51). Santiago de Chile: Editorial LOM.

Leiva, A. (2003). El tema de la participación de la psicología comunitaria en las políticas del nuevo trato. Polis Revista de la Universidad Bolivariana [online], 1(5). Recuperado el 15 de septiembre de 2016, de http:// polis.revues.org/6996

Montero, M. (2006). Teoría y práctica de la psicología comunitaria. La tensión entre comunidad y sociedad. Buenos Aires: Paidós.

Murphree, M. (2009). The strategic pillars of communal natural resource management: benefit, empowerment and conservation. Biodiversity Conservation, (18), 2551-2562.

Prilleltensky, I. (2003). The role of power in wellness, oppression, and liberation: The promise of psychopolitical validity. Journal of Community Psychology, 36(2), 116-136. doi: 10.1002/jcop.20225.

Rittel, H. y Webber, M. (1973). Dilemmas in general theory of planning. Policy Science, (4), 155-169.

Rivera, M., Velázquez, T. y Morote, R. (2014). Participación y fortalecimiento comunitario en un contexto posterremoto en Chincha, Perú. Psicoperspectivas, 13(2), 144-155.

Rodríguez, T. (2002). Proceso para la creatividad social. Madrid: El Viejo Topo. 
Sabatini, F., Sepúlveda, C. y Blanco, H. (2000). Participación ciudadana para enfrentar conflictos ambientales. Desafíos para el Sistema de Evaluación de Impacto Ambiental. Santiago de Chile: CIPMA.

Sánchez, E. (2000). Todos con la esperanza. Continuidad de la participación comunitaria. Caracas: CEPFHE, Universidad Central de Caracas.

Sandoval, J. (2003). Ciudadanía y juventud: el dilema entre la integración social y la diversidad cultural. Revista Última Década CIDPA, (19), 31-45.

Sapiains, R., Ugarte, A. M. y Rdz-Navarro, K. (2017). Tercera Encuesta Nacional de Medio Ambiente y Cambio Climático. Santiago de Chile: Ministerio del Medio Ambiente, Universidad de Chile, CR2.

Sepúlveda, C. y Bettati, B. (2005). El desastre ecológico del Santuario del río Cruces: Trizadura institucional y retroceso democrático. Ambiente y Desarrollo, 20(3), 62-68.

Tejerina, B. (2005). Movimientos sociales, espacio público y ciudadanía: Los caminos de la utopía. Revista Crítica de Ciencias Sociales, (72), 67-97.

Torquebiau, E. y Taylor, R. (2009). Natural resource management by rural citizens in developing countries: innovations still required. Biodiversity Conservation, (18), 2537-2550.

Turner, B. (1993). Contemporary problems in the theory of citizenship. En B. S. Turner (Ed.), Citizenship and social theory (pp. 1-18). Londres: Sage.

Villarroel, P. y Erlwein, E. (2007). Hacia una institucionalidad ambiental de segunda generación para Chile: La necesidad de un enfoque participativo, sinérgico e integrador. Revista Ambiente y Desarrollo, 23(3), $36-42$.

Wandersman, A. (1984). Citizen participation. En R. Price, S. R. Riger y A. Wandersman (Eds.), Psychology and community change (pp. 337-379). Dorsey: Homewood.

Webb, J. (2012). Climate change and society: The chimera of behaviour change technologies. Sociology, (46), $109-125$.

Wiesenfeld, E. y Sánchez, E. (2012). Participación, pobreza y políticas públicas: 3P que desafían la psicología ambiental comunitaria (el caso de los concejos comunales de Venezuela). Psychosocial Intervention, 21(3), 225-243.

Wilshusen, P., Brechin, S., Fortwangler, C. y West, P. (2002). Reinventing a square wheel: critique of a resurgent, protection paradigmyquot, in international biodiversity conservation. Society \& Natural Resources, 15(1), $17-40$. 